\title{
miR-134 suppresses the migration and invasion of non-small cell lung cancer by targeting ITGB1
}

\author{
QIN QIN ${ }^{1,2}$, FURONG WEI $^{3}$, JIANBO ZHANG $^{4}$ and BAOSHENG LI ${ }^{1}$ \\ ${ }^{1}$ Department of Radiation Oncology (Chest Section), Shandong Cancer Hospital and Institute, Shandong University, Jinan, \\ Shandong 250117; ${ }^{2}$ Department of Oncology, Jingzhou Central Hospital, The Second Clinical Medical College, \\ Yangtze University, Jingzhou, Hubei 434020; ${ }^{3}$ Institute of Basic Medicine, Shandong Academy of Medical Sciences, \\ School of Medicine and Life Sciences, University of Jinan-Shandong Academy of Medical Sciences, \\ Jinan, Shandong 250062; ${ }^{4}$ Department of Pathology, Shandong Cancer Hospital and Institute, \\ Shandong University, Jinan, Shandong 250117, P.R. China
}

Received May 31, 2016; Accepted June 29, 2016

DOI: $10.3892 /$ or.2017.5350

\begin{abstract}
Most cancer-related deaths are caused by the development of metastatic disease. Thus, investigation of the underlying mechanisms of metastasis is urgent to design more effective targeted drugs and to treat metastatic disease more effectively. MicroRNAs (miRNAs) have emerged as potential targets for cancer treatment. In the present study, we aimed to identify the roles of miR-134 in non-small cell lung cancer (NSCLC) cell migration and invasion. We demonstrated that overexpression of miR-134 inhibited migration and invasion of A549 and H1299 cells. Further mechanistic investigations revealed that miR-134 inhibited epithelial-to-mesenchymal transition (EMT) evidenced by upregulation of E-cadherin expression and downregulation of vimentin expression. Using luciferase assays, we identified integrin $\beta 1$ (ITGB1) as a direct target of miR-134. Performing RNAi and rescue experiments, we confirmed that miR-134 exerted its migratory and invasive suppressive role partly by downregulating ITGB1. Finally, an in vivo experiment also, to some extent, suggested that miR-134 may function as a suppressor of metastasis. Taken together, our findings suggest that miR-134 suppresses migration and invasion of NSCLC by targeting ITGB1.
\end{abstract}

\section{Introduction}

Lung cancer is the leading cause of cancer-related deaths both in China and worldwide (1), and metastasis is the major cause of cancer-related deaths (2). Mechanistically, metastasis is a complex process including tumor cell invasion of local vessels,

Correspondence to: Dr Baosheng Li, Department of Radiation Oncology (Chest Section), Shandong Cancer Hospital and Institute, Shandong University, 440 Jiyan Road, Jinan, Shandong 250117, P.R. China

E-mail: baoshli1963@163.com

Key words: non-small cell lung cancer, miR-134, migration, invasion, ITGB1 survival of circulating tumor cells, and colonization of tumor cells in distant organs. The success of metastasis depends on intrinsic properties of the tumor cells as well as interaction between tumor cells and tumor environmental factors (3). Investigation of the underlying mechanisms of tumor invasion and metastasis may help us predict tumor aggressiveness, tailor treatment according to metastatic potential and explore more effective therapeutic targets.

MicroRNAs (miRNAs) are small non-coding RNAs that regulate gene expression post-transcriptionally. In mammals, miRNAs are predicted to regulate the activity of $\sim 50 \%$ of all protein-coding genes (4). Deregulation of miRNAs has been implicated in various human diseases, particularly cancer (5). In addition to the regulation of tumor cell proliferation, differentiation and apoptosis, miRNAs also regulate tumor cell migration and invasion (6). Numerous miRNAs have been demonstrated to play an essential role in tumor metastasis (7-10).

miRNA-134 (miR-134) was initially identified as a brainspecific miRNA that is involved in synapse development (11). Subsequent investigations revealed its essential role in stem cell differentiation (12-14). Recently, numerous studies investigated the important functions of miR-134 in cancer, presenting inconsistent results (15-24). In non-small cell lung cancer (NSCLC), our previous data demonstrated that miR-134 inhibited tumor cell growth both in vitro and in vivo (25), but its role as a suppressor or promoter of metastasis is still controversial and warrants more investigation $(16,17,23)$. In the present study, we aimed to explore the role of miR-134 in NSCLC cell migration and invasion, and identify potential targets of miR-134.

\section{Materials and methods}

Cell culture. Human NSCLC cell lines (A549 and NCI-H1299) were purchased from the Type Culture Collection of the Chinese Academy of Sciences (Shanghai, China). Cells were cultured in RPMI-1640 medium supplemented with $10 \%$ fetal bovine serum (FBS), $100 \mathrm{U} / \mathrm{ml}$ penicillin and $100 \mu \mathrm{g} / \mathrm{ml}$ streptomycin and incubated in $5 \% \mathrm{CO}_{2}$ at $37^{\circ} \mathrm{C}$. 
Table I. Primers for real-time PCR.

\begin{tabular}{lll}
\hline Gene & \multicolumn{1}{c}{ Forward } & \multicolumn{1}{c}{ Reverse } \\
\hline ITGB1 & 5'-ATCCCAGAGGCTCCAAAGAT-3' & 5'-CCCCTGATCTTAATCGCAAA-3' \\
KRAS & 5'-TGGTGAGGGAGATCCGACAA-3' & 5'-AGGCATCATCAACACCCAGA-3' \\
FOXM1 & 5'-ATAGCAAGCGAGTCCGCATT-3' & 5'-AGCAGCACTGATAAACAAAGAAAGA-3' \\
GAPDH & 5'-CATGAGAAGTATGACAACAGCCT-3' & 5'-AGTCCTTCCACGATACCAAAGT-3' \\
\hline
\end{tabular}

ITGB1, integrin $\beta 1$.

miRNA and siRNA transfection. Cells were cultured to $40-50 \%$ confluency and transiently transfected with $50 \mathrm{nM}$ miRNA negative control (miR-NC), or miRNA mimics using HiPerFect Transfection Reagent (Qiagen, Germantown, MD, USA) according to the manufacturer's instructions. For RNAi experiments, siRNAs were also transfected using HiperFect Transfection Reagent at a final concentration of $50 \mathrm{nM}$. miRNA mimics and NC were purchased from GenePharma (Shanghai, China), and siRNAs were purchased from RiboBio Co., Ltd. (Guangzhou, China).

Transwell migration and invasion assays. For the migration assay, $2 \times 10^{4}$ transfected A549 or H1299 cells were seeded in the top chamber of the Transwell insert (BD Biosciences, Sparks, MD, USA). For the invasion assay, $3 \times 10^{5}$ transfected A549 or $1 \times 10^{5}$ transfected H1299 cells were seeded in the top chamber of the insert (BD Biosciences), which had previously been coated with $100 \mu \mathrm{l}$ diluted Matrigel (Corning, Corning, NY, USA). After $20-24 \mathrm{~h}$ of incubation at $37^{\circ} \mathrm{C}$, the cells that had migrated or invaded through the insert were fixed with $100 \%$ methanol, stained with $0.1 \%$ crystal violet and counted.

RNA extraction and quantitative real-time PCR. Total RNA containing miRNAs was isolated from cells using miRNeasy Mini kit (Qiagen). For single-stranded complementary DNA synthesis, 500 ng total RNA was reverse-transcribed using PrimeScript $^{\mathrm{TM}}$ RT reagent kit with gDNA Eraser (Takara, Dalian, China). Real-time PCR was performed using UltraSYBR Mixture (CWBio, Beijing, China) and LC 480 PCR System (Roche). Primers for integrin $\beta 1$ (ITGB1), KRAS, FOXM1 and GAPDH are shown in Table I. The expression levels of mRNA were normalized to the endogenous control GAPDH, using the $2^{-\Delta \Delta C t}$ method.

Western blotting. Cells were lysed using cell lysis buffer for western blot analysis and IP with protease inhibitor phenylmethylsulfonyl fluoride (PMSF) (Beyotime, Shanghai, China). Equal amounts of protein were separated by SDS-PAGE, and then transferred onto polyvinylidene difluoride membranes (Millipore, Billerica, MA, USA). Membranes were blocked with $5 \%$ non-fat milk in TBS containing $0.1 \%$ Tween-20, and then incubated with corresponding primary antibody. Primary antibodies against GAPDH (Abcam, Cambridge, UK) were used at a dilution of 1:10,000, against E-cadherin and vimentin (VIM) at a dilution of 1:1,000, and against ITGB1 (Abcam, Cambridge, UK) at a dilution of 1:2,000. Secondary antibodies conjugated with horseradish peroxidase (anti-mouse IgG and anti-rabbit $\mathrm{IgG}$ ) were used to detect primary antibodies. For HRP detection, an ECL chemiluminescence kit (CWBio) was used.

Luciferase reporter assays. The wild-type 3' untranslated region (3'UTR) of ITGB1 and its target-site mutant 3'UTR were amplified by PCR. The PCR products were then cloned into the XhoI/NotI site of the psiCHECK-2 dual-luciferase reporter plasmid (Promega, Madison, WI, USA). These vectors were named ITGB1-3'UTR, ITGB1-3'UTRm1, ITGB1-3'UTRm2 and ITGB1-3'UTRm1+2, respectively. To perform luciferase reporter assays, A549 and H1299 cells were plated into 96-well plates and co-transfected with the reporter vector and $50 \mathrm{nM}$ miR-NC or miR-134 mimics using Attractene Transfection Reagent (Qiagen). Forty-eight hours after transfection, Firefly and Renilla luciferase activities were measured using a Dual-Luciferase Reporter System (Promega).

Generation of A549 and H1299 cells stably expressing ITGBI. Lentiviral vector expressing ITGB1 or an empty lentiviral vector was purchased from GeneChem (Shanghai, China). Cell infection was performed following the manufacturer's protocol. Cells stably overexpressing ITGB1 or the empty vector were selected by puromycin $(2 \mu \mathrm{g} / \mathrm{ml})$. A549 and H1299 cells that stably expressed the empty vector or ITGB1 were designated A549-control and H1299-control, or A549-ITGB1 and H1299-ITGB1.

Functional rescue experiments. Rescue experiments were carried out to determine whether ITGB1 mediates the suppression effects on migration and invasion mediated by miR-134. A549-control, A549-ITGB1,H1299-control and H1299-ITGB1 cells were transfected with miR-NC or miR-134 mimics, and then the Transwell assays were performed.

Animal experiments. To establish a lung cancer xenograft model, $2 \times 10^{6}$ A549 cells in $100 \mu 1$ phosphate-buffered saline were subcutaneously injected intox the right hind limb of BALB/c nude mice [female, 4-5 weeks old, purchased from Beijing HFK Bioscience Co., Ltd. (Beijing China)]. After 10 days, when the diameter of the tumor reached $\sim 5-6 \mathrm{~mm}$, the nude mice were randomly divided into 2 groups $(n=4$ each). miR-134 agomir or negative control (NC) agomir (RiboBio Co., Ltd.) was then directly injected into the implanted tumor at the dose of $5 \mathrm{nmol} /$ mouse every 3 days for 5 times. Tumor volume (V) was monitored every 3 days since the first day of injection of agomir. Forty-eight hours after the last injection, 

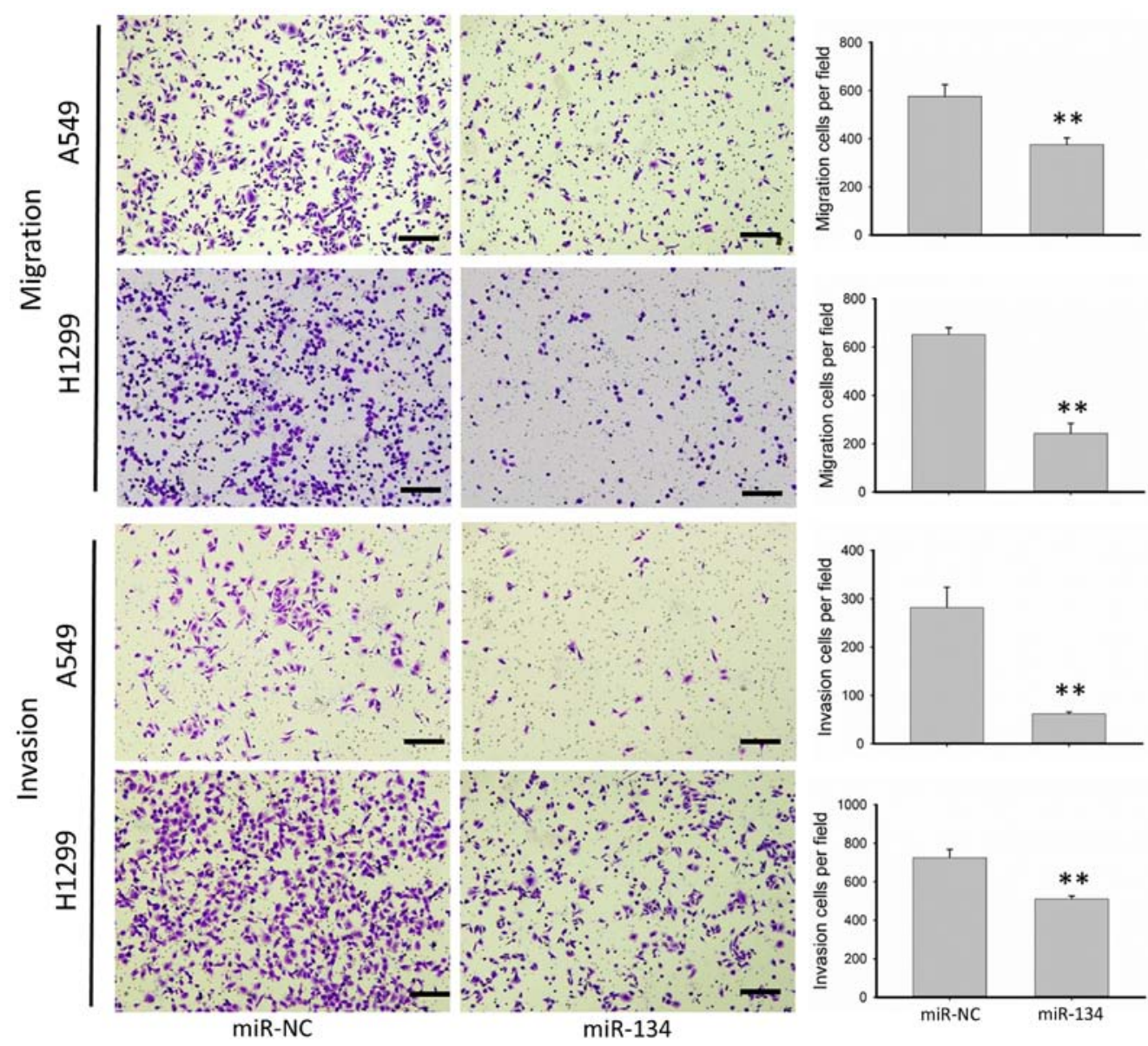

Figure 1. miR-134 inhibits cell migration and invasion of NSCLC. Transwell assays for A549 and H1299 cells transfected with miR-NC or miR-134 mimics. Representative images are shown with the quantification of 4 randomly selected fields; ${ }^{* *} \mathrm{P}<0.01$. Scale bars, $200 \mu \mathrm{m}$. NSCLC, non-small cell lung cancer.

animals were sacrificed and tumor tissues were resected. Lung tissues were also collected for inspection. Mice were manipulated and housed according to protocols approved by the Shandong Hospital Experimental Animal Care Commission.

Immunohistochemistry (IHC). Tumor tissues were fixed in formalin and embedded in paraffin. Sections (5- $\mu \mathrm{m}$ thick) were cut from the embedded tissues and mounted on polylysinecoated slides. Tumor sections were subjected to IHC staining for detection of E-cadherin, VIM and ITGB1. Briefly, sections were deparaffinized in xylene, rehydrated in gradient alcohol and treated with $0.3 \% \mathrm{H}_{2} \mathrm{O}_{2}$ for 15 min to quench endogenous peroxidase activity. Following antigen retrieval, sections were blocked in $10 \%$ normal serum with $1 \%$ BSA in TBS for $2 \mathrm{~h}$ at room temperature, and then incubated at $4^{\circ} \mathrm{C}$ overnight with the corresponding primary antibodies (E-cadherin, VIM and ITGB1; Abcam). Negative controls were incubated with the negative control antibody under the same condition. Next, the sections were incubated with biotinylated secondary antibody for $1 \mathrm{~h}$, followed by incubation with conjugated horseradish peroxidase streptavidin for $1 \mathrm{~h}$. Finally, the sections were incubated with diaminobenzidine and counterstained with hematoxylin.

Statistical analysis. Experiments were performed at least three times. Data were analyzed by Student's t-test when comparing two groups and by one-way ANOVA followed Bonferroni's post-test, when comparing more than two groups. A P-value $<0.05$ was considered to indicate a statistically significant result.

\section{Results}

miR-134 inhibits the migration and invasion of NSCLC cell lines. We chose NSCLC cell lines A549 and H1299 to investigate migration as well as invasion due to their innate aggressiveness. As shown in Fig. 1, miR-134 significantly inhibited the migration as well as the invasion of the A549 and H1299 cells. Overexpression of miR-134 in the A549 cells resulted in $\sim 45$ and $75 \%$ reduction in the number of migratory and invasive cells, respectively, while in the H1299 cells, migratory and invasive cells were decreased 60 and $30 \%$, respectively, in the cells transfected with miR-134 compared with cells transfected with the miR-NC.

miR-134 inhibits epithelial-to-mesenchymal transition (EMT), and downregulates ITGBI expression in NSCLC cell lines. EMT is an important process that increases the aggressiveness and metastatic potential of cancer cells. After revealing the suppressive roles of migration as well as invasion by miR-134, we aimed to ascertain whether or not this suppression was related to EMT inhibition. Thus, we detected the alteration 
A

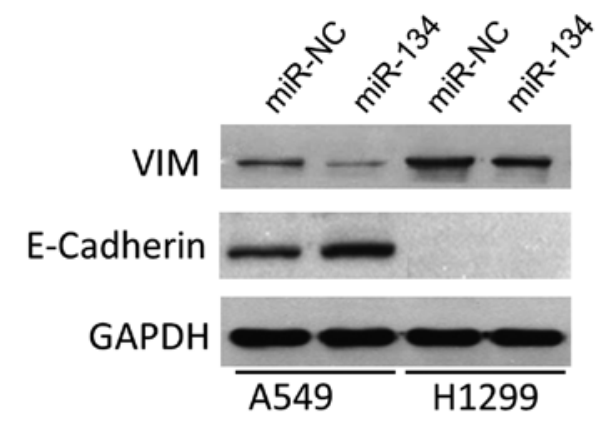

B

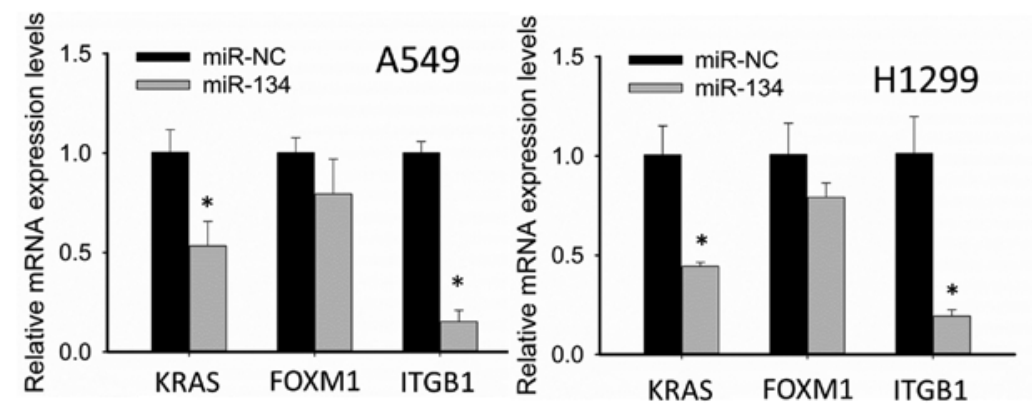

C

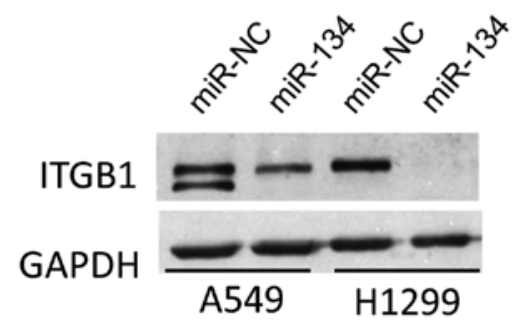

Figure 2. miR-134 inhibits EMT and downregulates ITGB1 expression in NSCLC cell lines. (A) Western blotting was performed to confirm the increased expression of E-cadherin in the A549 cells and the decreased expression of VIM in the A549 and H1299 cells after transfection of miR-134 mimics. No E-cadherin was detected by western blotting in the H1299 cells. (B) qRT-PCR was performed to investigate changes in the mRNA expression levels of KRAS, FOXM1 and ITGB1 in the A549 and H1299 cells. "P<0.05. (C) Western blotting was performed to confirm the downregulation of ITGB1 expression in the A549 and H1299 cells after transfection with the miR-134 mimics. EMT, epithelial-to-mesenchymal transition; ITGB1, integrin $\beta 1$; NSCLC, non-small cell lung cancer; VIM, vimentin.

in expression of two essential molecules participating in EMT: E-cadherin (epithelial marker) and VIM (mesenchymal marker). As shown in Fig. 2A, in the A549 cells, miR-134 increased the expression of epithelial marker E-cadherin, and decreased the expression of mesenchymal marker VIM; in H1299 cells, there was no E-cadherin detected, while the expression of VIM was significantly suppressed. These data indicated that miR-134 inhibited EMT in NSCLC cells, which may be partly responsible for suppression of migration and invasion by miR-134.

Although we demonstrated that miR-134 inhibited EMT of NSCLC cells, neither E-cadherin nor VIM was found to be the predicted target of miR-134. Thus, we were motivated to identify potential targets. Through integrating both target prediction as well as literature review, we focused our attention on the following 3 pertinent oncogenes, KRAS, FOXM1 and ITGB1, which have been confirmed as authentic targets of miR-134. qRT-PCR suggested that ITGB1 showed the most significant decrease after miR-134 transfection (Fig. 2B). Therefore, we chose ITGB1 for further western blotting validation. As shown in Fig. 2C, western blotting confirmed the downregulation of ITGB1.
ITGB1 is a direct target of miR-134 in NSCLC cell lines. To determine whether downregulation of the ITGB1 expression levels are due to direct targeting of miR-134 to the ITGB13'UTR, we constructed reporter vectors containing wild-type (ITGB1-3'UTR) and target-site mutant (ITGB1-3'UTRm1, ITGB1-3'UTRm2 and ITGB1-3'UTRm1+2) 3'UTR of ITGB1 respectively to perform luciferase reporter assay in the A549 and H1299 cells (Fig. 3A). Compared with miR-NC, co-transfection of miR-134 and ITGB1-3'UTR showed significantly decreased luciferase activity, whereas co-transfection of miR-134 and ITGB1-3'UTRm1+2 did not result in significant reduction in luciferase activity. Co-transfection of miR-134 and ITGB1-3'UTRm1 or ITGB1-3'UTRm2 also resulted in reduced luciferase activity; however, the degree of reduction was less than the co-transfection of miR-134 and ITGB13'UTR (Fig. 3B). These results indicated that both target sites in ITGB1-3'UTR are targeted by miR-134, and the second target site is more potent than the first one.

ITGBI mediates the suppressive effect on migration and invasion by miR-134 in NSCLC cell lines. To determine whether miR-134 exerts its suppressive functions on migration and 


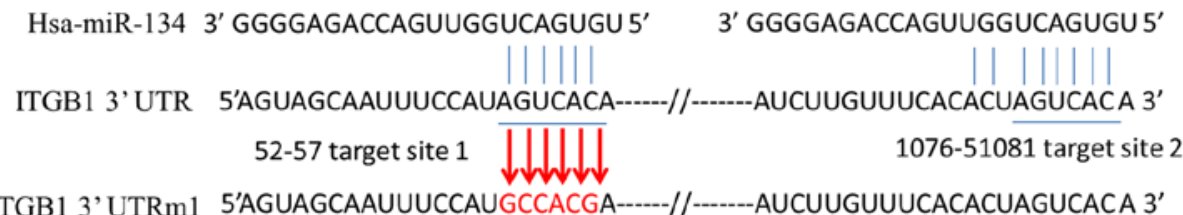

ITGB1 3'UTRm1 5'AGUAGCAAUUUCCAUGCCACGA-----//------AUCUUGUUUCACACUAGUCACA 3'

ITGB1 3'UTRm2 5'AGUAGCAAUUUCCAUAGUCACA-----//------AUCUUGUUUCACACUGCCACGA 3'

ITGB1 3'UTRm1+2 5'AGUAGCAAUUUCCAUGCCACGA-----//-------AUCUUGUUUCACACUGCCACG A 3'

B
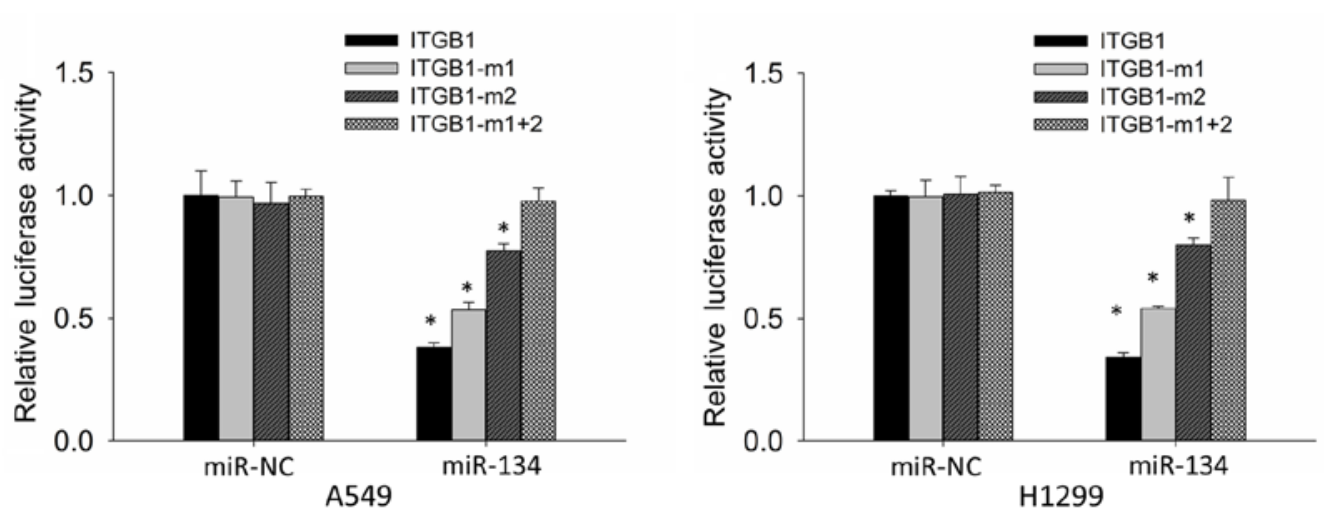

Figure 3. Luciferase reporter assays confirm that ITGB1 is a direct target of mR-134 in the NSCLC cell lines. (A) Predicted miR-134 target sites in ITGB1 3'UTR and the structure of reporter vectors. (B) Luciferase reporter assays were performed in the A549 and H1299 cells by co-transfection of miR-NC or miR-134 mimics with reporter vectors ITGB1-3'UTR or ITGB1-3'UTRm1 or ITGB1-3'UTRm2 or ITGB1-3'UTRm1+2. Experiments were performed with 3 replicates and data are shown as mean $\pm \mathrm{SD} ;{ }^{*} \mathrm{P}<0.05$. ITGB1, integrin $\beta 1$; NSCLC, non-small cell lung cancer.

invasion through downregulation of ITGB1, we performed RNAi and rescue experiments. RNAi experiment confirmed that knockdown of ITGBI significantly inhibited EMT, cell migration and invasion, mimicking the phenotype of miR-134 overexpression (Fig. 4A and B). In the functional rescue experiments, we constructed ITGB1-overexpressing A549 and H1299 cells using a lentiviral vector (A549-ITGB1 and H1299-ITGB1), in which the 3'UTR of ITGB1 was missing. ITGB1 overexpression was confirmed by western blotting. While miR-134 inhibited ITGB1 expression in the A549 and H1299 cells transfected with the lentiviral-vector control (A549-control and H1299-control), no significant downregulation of ITGB1 was detected in the A549-ITGB1 and H1299-ITGB1 cells (Fig. 4C). In the A549-ITGB1 and H1299ITGB1 cells, the suppressive effects on tumor migration and invasion of miR-134 were abolished (Fig. 4D), suggesting that ITGB1 is a functional target of miR-134.

miR-134 inhibits EMT and downregulates ITGB1 expression in an A549 lung cancer xenograft model. To investigate whether miR-134 inhibits NSCLC cell metastasis in vivo, we constructed an A549 lung cancer xenograft model, and treated the mice with an intro-tumor injection of miR-134 agomir or NC agomir. miR-134 agomir treatment significantly inhibited A549 xenograft growth (data not shown). However, at the time of sacrifice, no metastasis was detected in lung tissues, thus we performed IHC detection of E-cadherin, VIM and ITGB1 as a surrogate to assess the metastatic potential of the tumor cells. As shown in Fig. 5, the expression level of E-cadherin was increased, while the expression levels of
VIM and ITGB1 were decreased in the tumors injected with the miR-134 agomir compared to these levels in the tumors injected with NC agomir. These data indicated that miR-134 increased E-cadherin and decreased VIM expression in vivo, which was correlated with decreased EMT and aggressiveness, and downregulated ITGB1 expression, which also suggested decreased invasion in vivo.

\section{Discussion}

Most cancer-related deaths are caused by the development of metastatic disease, since patients with systemically disseminated disease hold more tumor burden and tumor cells possess more comprehensive heterogeneity that render them more refractory to currently available treatments (2). Considering the enormous discrepancies of metastatic potential even among patients with the same tumor type, it is worthwhile to explore any possible approach that may reduce tumor metastasis.

miRNAs are key regulators in the initiation and development of cancer. They function as tumor suppressors or oncogenes and are potential targets for cancer treatment $(6,26)$. In the present study, we identified that miR-134 is a suppressor of metastasis in NSCLC. Our results indicated that miR-134 inhibited A549 and H1299 cell migration and invasion, which is consistent with two previous studies (17,23), and inconsistent with another study (16). As these studies and ours all investigated the roles of miR-134 in A549 cells, the reasons for such an inconsistence are difficult to explain.

EMT is a pivotal process that enables tumor cells to acquire a more migratory and invasive mesenchymal 
A

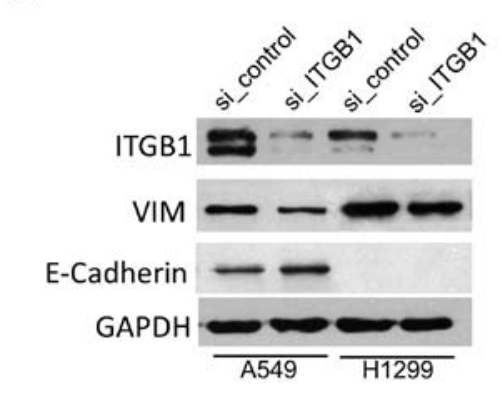

C

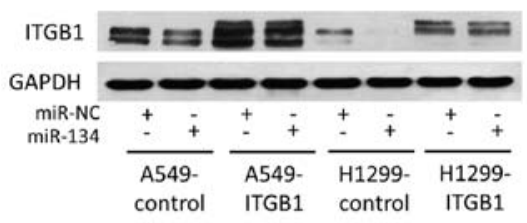

B
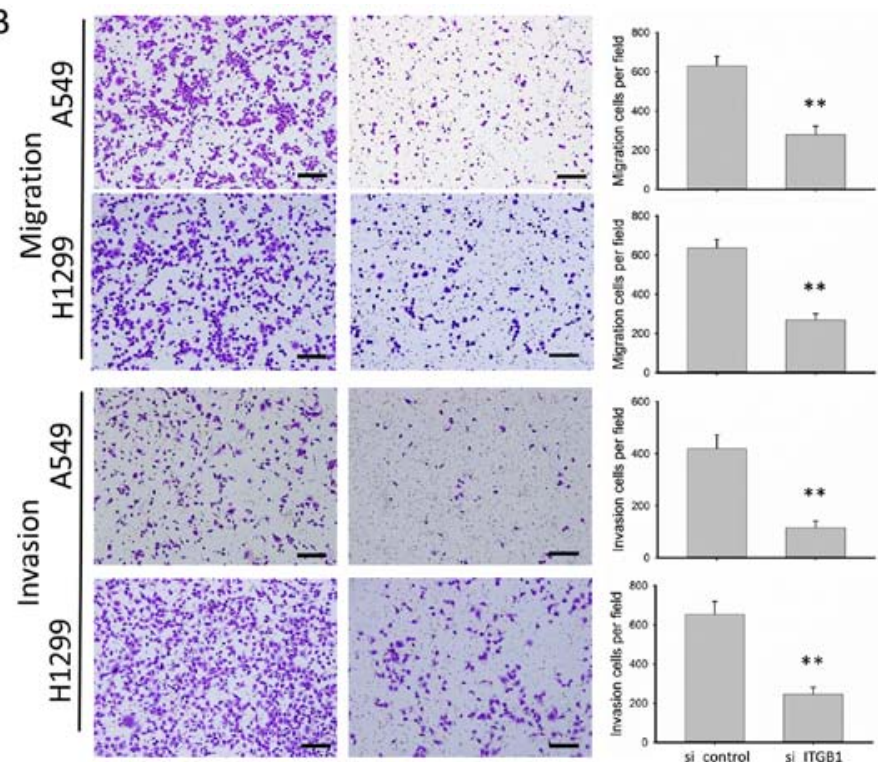

si_control
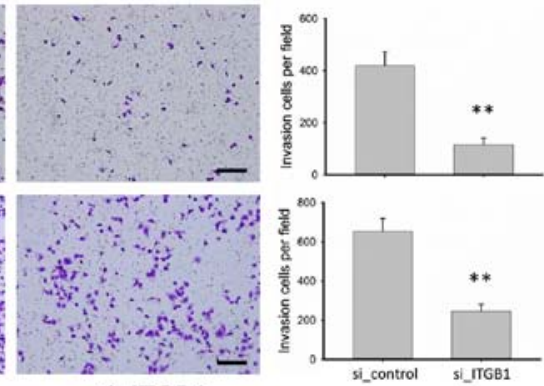

D
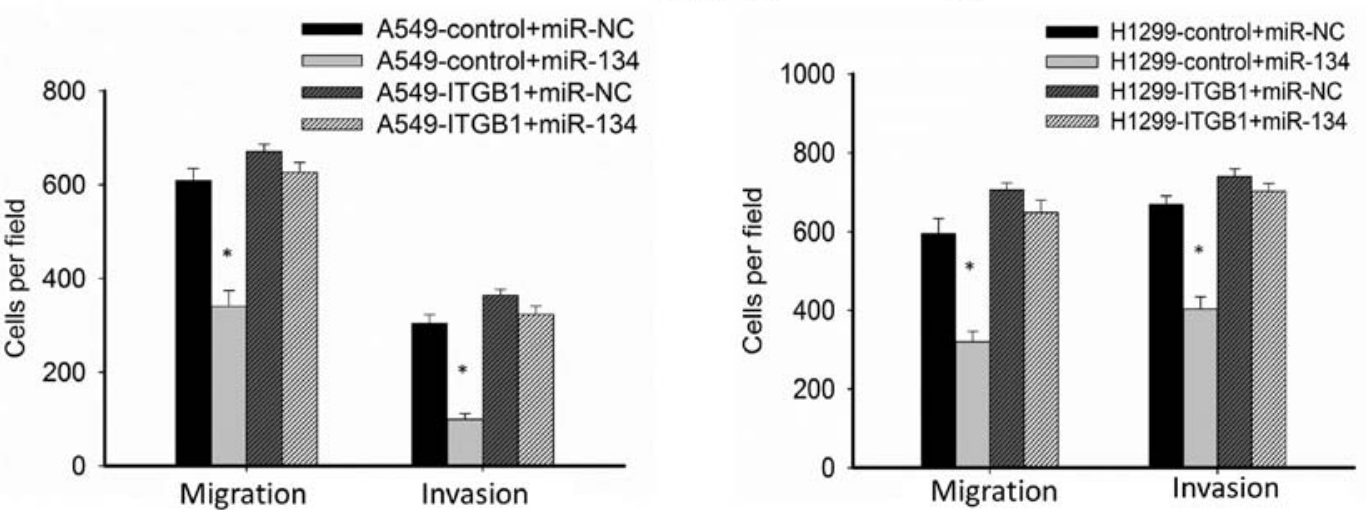

Figure 4. ITGB1 mediates the suppressive role of miR-134 on the migration and invasion of the NSCLC cell lines. (A) Western blotting was performed to confirm the knockdown of ITGB1 expression in the A549 and H1299 cells after transfection with si_ITGB1. Alterations in E-cadherin and VIM expression are also shown. (B) Transwell assays were performed in the A549 and H1299 cells after transfection with si_control or si_ITGB1, suggesting that knockdown of ITGB1 suppressed cell migration and invasion. (C) Western blotting was performed to detect ITGB1 expression in the A549-control, A549-ITGB1, H1299control and H1299-ITGB1 cells after transfection with miR-NC or miR-134. (D) Transwell assays suggested that ITGB1 overexpression partly rescued the suppressive effect of miR-134 on cell migration and invasion. Results are shown as mean $\pm \mathrm{SD} ;{ }^{*} \mathrm{P}<0.05,{ }^{* *} \mathrm{P}<0.01$. Scale bars, $200 \mu \mathrm{m}$. ITGB1, integrin $\beta 1$; NSCLC, non-small cell lung cancer; VIM, vimentin.

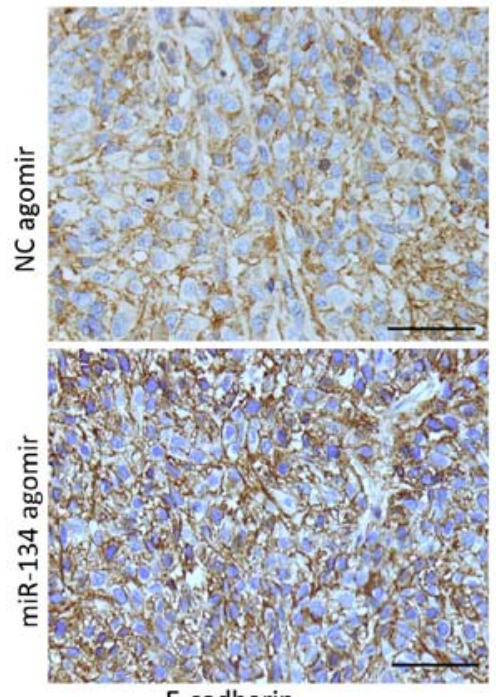

E-cadherin
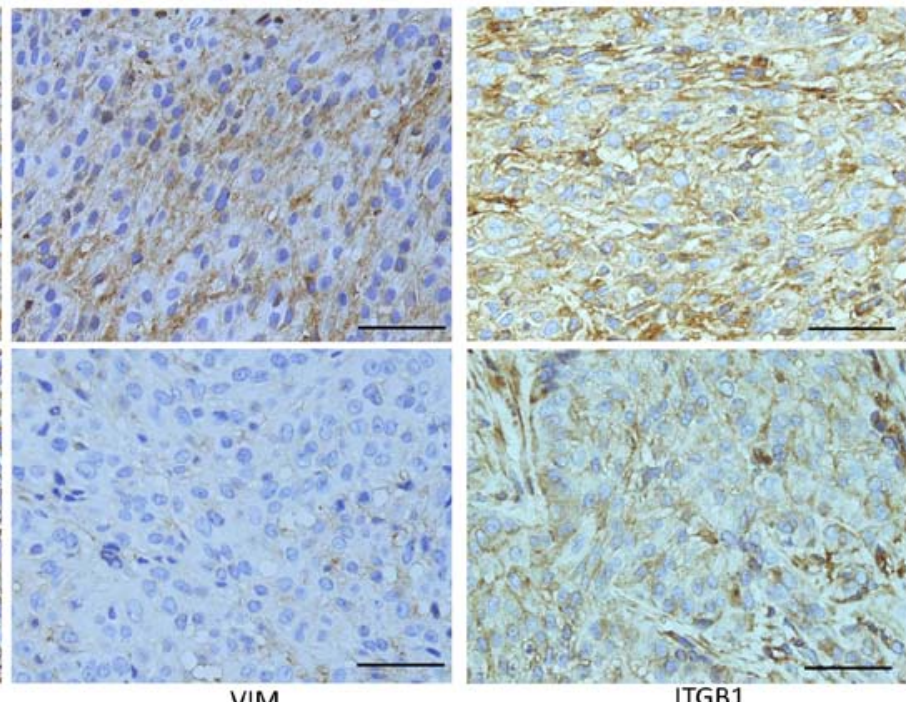

ITGB1

Figure 5. miR-134 inhibits EMT and downregulates ITGB1 expression in an A549 lung cancer xenograft model. IHC staining was used to detect the expression of E-cadherin, VIM and ITGB1 in the xenograft sections. Scale bars, $50 \mu \mathrm{m}$. EMT, epithelial-to-mesenchymal transition; ITGB1, integrin $\beta 1$; VIM, vimentin. 
phenotype, during which tumor cells exhibit downregulation of the expression of epithelial proteins such as E-cadherin, and upregulation of the expression of mesenchymal proteins such as N-cadherin and VIM (27). We found in the present study that miR-134 increased the expression of E-cadherin and decreased the expression of VIM, suggesting that miR-134 inhibits or reverses the process of EMT. These results were similar to one study (17), yet different to another (16). Still, the underlying reasons for such an apparent discordance are unknown.

To identify potential targets of miR-134 that may be responsible for its suppression of migration and invasion, we selected 3 molecules: KRAS, FOXM1 and ITGB1, which were confirmed to be authentic targets in other cancers [KRAS $(19,21,24)$ and ITGB1 (22)] or NSCLC [FOXM1 (17)], for validation. We found that the expression level of ITGB1 showed the most significant decline after transfection of miR-134. Considering such a downregulation of ITGB1 and the importance of ITGB1 in lung cancer metastasis (28), we chose ITGB1 for further testing.

ITGB1 is an essential subunit of the integrin family which mediates cell-extracellular matrix (ECM) adhesion and signaling that affect diverse cellular processes, including proliferation, apoptosis, migration, invasion and survival (29). Elevated expression or activation of ITGB1 signaling not only correlates with poor prognosis in some solid tumors including lung cancer $(28,30,31)$, but also confers resistance to chemotherapy $(32)$, radiotherapy $(33,34)$ and targeted therapy (35-37), which renders ITGB1 as a potential target. Strategies that target ITGB1 have been extensively investigated in preclinical and clinical models, showing promising efficacy (38-41). In the present study, we showed that ITGB1 could be suppressed by miR-134 in NSCLC. Downregulation of ITGB1 by miR-134 or siRNA both suppressed NSCLC cell migration and invasion. However, miR-134 may be more advantageous than siRNA as miRNAs may target a broad set of oncogenes simultaneously (26).

During the development of metastasis, regulation of cell to cell adhesion and cell to extracellular matrix (EMC) adhesion is essential for cancer cell migration and invasion (2). In the present study, we demonstrated that miR-134 increased E-cadherin expression, which is a key mediator of cell to cell adhesion (27), thereby enhancing cell to cell adhesion and reducing cancer cell mobility. Meanwhile, miR-134 decreased ITGB1 expression, which mediates cell to EMC adhesion (29), thereby impairing cell to ECM adhesion and suppressing the invasion of cancer cells.

In conclusion, we demonstrated that miR-134 downregulated ITGB1 expression and inhibited EMT, resulting in suppressed migration and invasion of NSCLC both in vitro and in vivo. ITGB1 is a direct functional target of miR-134. Therefore, miR-134 replacement may be an approach to target ITGB1 and ITGB1-associated metastasis in NSCLC.

\section{Acknowledgements}

The present study was supported by the National Natural Science Foundation of China (grant nos. 81272501 and 81530060), and the Taishan Scholars Program of Shandong Province, China (grant no. ts20120505).

\section{References}

1. Chen W, Zheng R, Baade PD, Zhang S, Zeng H, Bray F, Jemal A $\mathrm{Yu}$ XQ and He J: Cancer statistics in China, 2015. CA Cancer J Clin 66: 115-132, 2016.

2. Valastyan S and Weinberg RA: Tumor metastasis: Molecular insights and evolving paradigms. Cell 147: 275-292, 2011.

3. Chaffer CL and Weinberg RA: A perspective on cancer cell metastasis. Science 331: 1559-1564, 2011.

4. Krol J, Loedige I and Filipowicz W: The widespread regulation of microRNA biogenesis, function and decay. Nat Rev Genet 11: 597-610, 2010.

5. Lu J, Getz G, Miska EA, Alvarez-Saavedra E, Lamb J, Peck D, Sweet-Cordero A, Ebert BL, Mak RH, Ferrando AA, et al: MicroRNA expression profiles classify human cancers. Nature 435: 834-838, 2005.

6. Kasinski AL and Slack FJ: Epigenetics and genetics. MicroRNAs en route to the clinic: Progress in validating and targeting microRNAs for cancer therapy. Nat Rev Cancer 11: 849-864, 2011.

7. Zhang H, Li Y and Lai M: The microRNA network and tumor metastasis. Oncogene 29: 937-948, 2010.

8. Cui R, Meng W, Sun HL, Kim T, Ye Z, Fassan M, Jeon YJ, Li B, Vicentini C, Peng Y, et al: MicroRNA-224 promotes tumor progression in nonsmall cell lung cancer. Proc Natl Acad Sci USA 112: E4288-E4297, 2015.

9. Chang RM, Xu JF, Fang F, Yang H and Yang LY: MicroRNA-130b promotes proliferation and EMT-induced metastasis via PTEN/ p-AKT/HIF-1 $\alpha$ signaling. Tumour Biol 37: 10609-10619, 2016.

10. Gregory PA, Bert AG, Paterson EL, Barry SC, Tsykin A, Farshid G, Vadas MA, Khew-Goodall Y and Goodall GJ: The miR-200 family and miR-205 regulate epithelial to mesenchymal transition by targeting ZEB1 and SIP1. Nat Cell Biol 10: 593-601, 2008.

11. Schratt GM, Tuebing F, Nigh EA, Kane CG, Sabatini ME, Kiebler $M$ and Greenberg ME: A brain-specific microRNA regulates dendritic spine development. Nature 439: 283-289, 2006.

12. Poitz DM, Stölzel F, Arabanian L, Friedrichs J, Docheva D, Schieker M, Fierro FA, Platzbecker U, Ordemann R, Werner C, et al: MiR-134-mediated $\beta 1$ integrin expression and function in mesenchymal stem cells. Biochim Biophys Acta 1833: 3396-3404, 2013.

13. Tay Y, Zhang J, Thomson AM, Lim B and Rigoutsos I: MicroRNAs to Nanog, Oct4 and Sox 2 coding regions modulate embryonic stem cell differentiation. Nature 455: 1124-1128, 2008.

14. Tay YM, Tam WL, Ang YS, Gaughwin PM, Yang H, Wang W, Liu R, George J, Ng HH, Perera RJ, et al: MicroRNA-134 modulates the differentiation of mouse embryonic stem cells, where it causes post-transcriptional attenuation of Nanog and LRH1. Stem Cells 26: 17-29, 2008.

15. Chen T, Gao F, Feng S, Yang T and Chen M: MicroRNA-134 regulates lung cancer cell $\mathrm{H} 69$ growth and apoptosis by targeting WWOX gene and suppressing the ERK1/2 signaling pathway. Biochem Biophys Res Commun 464: 748-754, 2015.

16. Kitamura K, Seike M, Okano T, Matsuda K, Miyanaga A, Mizutani H, Noro R, Minegishi Y, Kubota K and Gemma A: MiR-134/487b/655 cluster regulates TGF- $\beta$-induced epithelial-mesenchymal transition and drug resistance to gefitinib by targeting MAGI2 in lung adenocarcinoma cells. Mol Cancer Ther 13: 444-453, 2014.

17. Li J, Wang Y, Luo J, Fu Z, Ying J, Yu Y and Yu W: miR-134 inhibits epithelial to mesenchymal transition by targeting FOXM1 in non-small cell lung cancer cells. FEBS Lett 586: 3761-3765, 2012.

18. Liu CJ, Shen WG, Peng SY, Cheng HW, Kao SY, Lin SC and Chang KW: $m i R-134$ induces oncogenicity and metastasis in head and neck carcinoma through targeting WWOX gene. Int J Cancer 134: 811-821, 2014.

19. Liu Y, Zhang M, Qian J, Bao M, Meng X, Zhang S, Zhang L, Zhao R, Li S, Cao Q, et al: miR-134 functions as a tumor suppressor in cell proliferation and epithelial-to-mesenchymal Transition by targeting KRAS in renal cell carcinoma cells. DNA Cell Biol 34: 429-436, 2015.

20. Niu CS, Yang Y and Cheng CD: MiR-134 regulates the proliferation and invasion of glioblastoma cells by reducing Nanog expression. Int J Oncol 42: 1533-1540, 2013. 
21. Yin C, Wang PQ, Xu WP, Yang Y, Zhang Q, Ning BF, Zhang PP, Zhou WP, Xie WF, Chen WS, et al: Hepatocyte nuclear factor-4a reverses malignancy of hepatocellular carcinoma through regulating miR-134 in the DLK1-DIO3 region. Hepatology 58: 1964-1976, 2013.

22. Zha R, Guo W, Zhang Z, Qiu Z, Wang Q, Ding J, Huang S, Chen T, Gu J, Yao M, et al: Genome-wide screening identified that miR-134 acts as a metastasis suppressor by targeting integrin $\beta 1$ in hepatocellular carcinoma. PLoS One 9: e87665, 2014.

23. Zhang X, Wang H, Zhang S, Song J, Zhang Y, Wei X and Feng Z: MiR-134 functions as a regulator of cell proliferation, apoptosis, and migration involving lung septation. In Vitro Cell Dev Biol Anim 48: 131-136, 2012.

24. Zhang Y, Kim J, Mueller AC, Dey B, Yang Y, Lee DH, Hachmann J, Finderle S, Park DM, Christensen J, et al: Multiple receptor tyrosine kinases converge on microRNA-134 to contro KRAS, STAT5B, and glioblastoma. Cell Death Differ 21 720-734, 2014

25. Qin Q, Wei F, Zhang J, Wang X and Li B: miR-134 inhibits non-small cell lung cancer growth by targeting the epidermal growth factor receptor. J Cell Mol Med 20: 1974-1983, 2016

26. Chen Y, Gao DY and Huang L: In vivo delivery of miRNAs for cancer therapy: Challenges and strategies. Adv Drug Deliv Rev 81: 128-141, 2015

27. Lamouille S, Xu J and Derynck R: Molecular mechanisms of epithelial-mesenchymal transition. Nat Rev Mol Cell Biol 15: 178-196, 2014

28. Oshita F, Kameda Y, Hamanaka N, Saito H, Yamada K, Noda K and Mitsuda A: High expression of integrin betal and p53 is a greater poor prognostic factor than clinical stage in small-cell lung cancer. Am J Clin Oncol 27: 215-219, 2004.

29. Desgrosellier JS and Cheresh DA: Integrins in cancer: Biological implications and therapeutic opportunities. Nat Rev Cancer 10 9-22, 2010

30. Nikkola J, Vihinen P, Vlaykova T, Hahka-Kemppinen M, Heino J and Pyrhönen S: Integrin chains betal and alphav as prognostic factors in human metastatic melanoma. Melanoma Res 14: 29-37, 2004.

31. Yao ES, Zhang H, Chen YY, Lee B, Chew K, Moore D and Park C: Increased beta1 integrin is associated with decreased survival in invasive breast cancer. Cancer Res 67: 659-664, 2007.
32. Damiano JS: Integrins as novel drug targets for overcoming innate drug resistance. Curr Cancer Drug Targets 2: 37-43, 2002.

33. Cordes N, Seidler J, Durzok R, Geinitz H and Brakebusch C: Beta1-integrin-mediated signaling essentially contributes to cell survival after radiation-induced genotoxic injury. Oncogene 25 : 1378-1390, 2006

34. Park CC, Zhang HJ, Yao ES, Park CJ and Bissell MJ: Beta1 integrin inhibition dramatically enhances radiotherapy efficacy in human breast cancer xenografts. Cancer Res 68: 4398-4405, 2008.

35. Huang C, Park CC, Hilsenbeck SG, Ward R, Rimawi MF, Wang YC, Shou J, Bissell MJ, Osborne CK and Schiff R: $\beta 1$ integrin mediates an alternative survival pathway in breast cancer cells resistant to lapatinib. Breast Cancer Res 13: R84, 2011.

36. Kanda R, Kawahara A, Watari K, Murakami Y, Sonoda K, Maeda M, Fujita H, Kage M, Uramoto H, Costa C, et al: Erlotinib resistance in lung cancer cells mediated by integrin $\beta 1 /$ Src/Akt-driven bypass signaling. Cancer Res 73: 6243-6253, 2013.

37. Jahangiri A, Aghi MK and Carbonell WS: $\beta 1$ integrin: Critical path to antiangiogenic therapy resistance and beyond. Cancer Res 74: 3-7, 2014.

38. Park CC, Zhang H, Pallavicini M, Gray JW, Baehner F, Park CJ and Bissell MJ: Beta1 integrin inhibitory antibody induces apoptosis of breast cancer cells, inhibits growth, and distinguishes malignant from normal phenotype in three dimensional cultures and in vivo. Cancer Res 66: 1526-1535, 2006.

39. Bhaskar V, Zhang D, Fox M, Seto P, Wong MH, Wales PE, Powers D, Chao DT, Dubridge RB and Ramakrishnan V: A function blocking anti-mouse integrin alpha5beta1 antibody inhibits angiogenesis and impedes tumor growth in vivo. J Transl Med 5: 61, 2007.

40. Eke I, Zscheppang K, Dickreuter E, Hickmann L, Mazzeo E, Unger $K$, Krause $M$ and Cordes N: Simultaneous $\beta 1$ integrin-EGFR targeting and radiosensitization of human head and neck cancer. J Natl Cancer Inst 107: dju419, 2015.

41. Ricart AD, Tolcher AW, Liu G, Holen K, Schwartz G, Albertini M, Weiss G, Yazji S, Ng C and Wilding G: Volociximab, a chimeric monoclonal antibody that specifically binds $\alpha_{5} \beta_{1}$ integrin: A phase I, pharmacokinetic, and biological correlative study. Clin Cancer Res 14: 7924-7929, 2008. 